\title{
The quantitative proteomic analysis provides insight into the effects of drought stress in maize
}

\author{
H.-J. LI', Y.-F. WANG ${ }^{\dagger}$, C.-F. ZHAO, M. YANG, G.-X. WANG, and R.-H. ZHANG ${ }^{+}$ \\ College of Agronomy, Northwest A\&F University, Yangling, 712100 Shaanxi, China
}

\begin{abstract}
Drought stress is one of the major environmental factors that limit maize yield in agriculture. However, few studies have analyzed how proteins respond to different degrees of drought at the proteome level. In this study, physiological characteristics and comparative tandem mass tag proteomics were used to analyze the responses of maize seedlings to mild and severe drought stresses in pot experiments. A total of 104 and 464 proteins were differentially expressed under mild and severe drought, respectively, but only 30 proteins were overlapped. Further Gene Ontology enrichment analysis showed maize can adapt to mild drought by activating antioxidant system and photorespiration. Under severe drought stress, photosystem and protein synthesis-related proteins were downregulated indicating severe drought damaged the photosynthetic apparatus. The plant biomass under drought stress was also reduced sharply compared to control. Taken together, our study provides insights into proteomic information of maize leaves under increasing drought stress.
\end{abstract}

Keywords: comparative proteomics; maize; mild drought; photosynthesis; severe drought.

\section{Introduction}

Drought stress is one of the detrimental environmental factors that limit plant productivity in agriculture $(\mathrm{Zhu}$ 2016). Plant sensitivity to drought is a complex phenomenon and depends on numerous factors, such as the growth stage of the plant, duration, and severity of stress (Zhu 2002). How plants adapt to adverse environments is a fundamental biological question. Accumulating knowledge shows that plants have evolved complex physiological and molecular mechanisms to respond to drought (Zhou et al. 2015, Habibi 2018). Under the condition of water deficiency, plants respond to drought stress by controlling leaf rolling, stomatal aperture, and density at the morphological level. Physiological traits, such as accumulation of compatible solutes (e.g., glycine betaine and proline), enhancement of antioxidant enzymatic systems (e.g., peroxidase and superoxide dismutase), biosynthesis of wax, are generally considered references alleviating oxidative damage (Salehi-Lisar and Bakhshayeshan-Agdam 2016). At the molecular level, the plant responds to drought stress by modifying its mechanism and physiology, for example, the induction of regulatory and functional genes, signal transduction, and activation of transcriptional regulators

\section{Highlights}

- Maize can adapt to mild drought by activating antioxidant system and photorespiration

- Severe drought damaged the photosynthetic apparatus and impaired the growth of maize.

- The further desiccation will cause the irreversible damage in maize
Received 18 July 2020

Accepted 13 November 2020

Published online 11 December 2020

${ }^{+}$Corresponding author

e-mail: zhangrenhe@nwsuaf.edu.cn

Abbreviations: APX - ascorbate peroxidase; CAT - catalase; $C_{\mathrm{i}}$ - intercellular $\mathrm{CO}_{2}$ concentration; DEPs - differentially expressed proteins; GO - Gene Ontology; $g_{s}$ - stomatal conductance; GST - glutathione S-transferase; HSPs - heat shock proteins; KEGG Kyoto Encyclopedia of Genes and Genomes; $P_{\mathrm{N}}$ - net photosynthetic rate; POD - peroxidase; ROS - reactive oxygen species; SOD - superoxide dismutase; TMT - tandem mass tag.

Acknowledgments: This work was supported by the National Key Research and Development Program of China (2017YFD0300304) and the Modern Agricultural Industry Technology Research System (CARS-02-53).

These authors contributed equally to the work.

Conflict of interest: The authors declare that they have no conflict of interest. 
(Anjum et al. 2011). However, knowledge about comprehensive regulatory mechanisms of plant response to drought stress is limited.

Recent rapid advances of high-throughput sequencing technology have enabled analyzing the complex mechanisms of plant response to stress at transcriptome and proteome levels (Chen et al. 2019, Dong et al. 2019). For instance, transcriptomic analyses have been widely used to identify differentially expressed genes in drought conditions (Huang et al. 2014, Solis et al. 2016). However, the transcript abundances usually do not correlate well with protein abundance and physiological performance. A proteomics approach is a powerful tool for global characterization of protein dynamics. Because protein is not only the implementer of gene encoding function but also controls the metabolic process. Proteomics has been successfully used to study drought stress responses in a wide range of crops through the identification of regulated proteins, including wheat (Triticum aestivum) (Cui et al. 2019), maize (Zea mays) (Jiang et al. 2019), soybean (Glycine max) (Liu et al. 2018a), rice (Oryza sativa) (Wu et al. 2019). These identified proteins are mainly related to transcription, protein synthesis, folding and degradation, stress and defense, photosynthesis, carbohydrate and energy metabolism, signaling, cell structure and cycle, membrane and transport, secondary metabolism. Therefore, a better understanding of crop drought tolerance, especially changes to the proteome under prolonged water deficit, can provide new insights for the analysis of complex mechanisms.

Maize as a widely cultivated food crop in the arid and semiarid regions, such as Northwest China, is often affected by moisture deficit or drought, particularly at the seedling stage, thereby threatening germination and seedling growth (Liu et al. 2018b). Analyzing the proteome profiles of maize under various intensity drought stress conditions will increase our understanding of the complex regulatory network and response mechanisms to drought stress. Thus far, most studies have mainly focused on problems associated with cultivation, physiological characteristics, genetics, and a single time point analysis of drought stress (Jogaiah et al. 2013, Kim et al. 2015, Zheng et al. 2020). Due to the technological limitations of two-dimensional gel electrophoresis (2-DE) in proteomics, tandem mass tag (TMT)-based proteomic analysis of maize leaves in response to different drought stress still has rarely been reported.

In the present study, we performed TMT-based comparative quantitative proteomics analysis to reveal the changes in proteomic profiles of maize leaves under mild and severe drought stress. Through bioinformatics analysis, 104 and 464 differentially expressed proteins (DEPs) were identified in mild and severe drought, respectively. We also characterized various physiological parameters, including leaf gas exchange, plant biomass, and antioxidant activities [superoxide dismutase (SOD), peroxidase (POD), catalase (CAT), and ascorbate peroxidase (APX)]. Then, the qRTPCR results were used to validate the expression changes in proteins. This result can enhance our understanding of the molecular basis of drought tolerance and would be used for a future breeding program in maize.

\section{Materials and methods}

Plant materials and drought treatments: The maize (Zea mays L.) hybrid Shaanke 9 was used as plant material. Uniformly germinated seeds were directly sown into plastic pots (26 cm diameter $\times 38 \mathrm{~cm}$ high) filled with $18 \mathrm{~kg}$ of air-dried soil, composed of $1.62 \%$ organic matter and $0.064 \%$ total nitrogen. The seedlings were grown in a greenhouse with a day/night temperature of $26 / 18^{\circ} \mathrm{C}$, relative humidity of $60 \%$, and natural light at Northwest A\&F University, Yangling $\left(34^{\circ} 283^{\prime} \mathrm{N}, 108^{\circ} 067^{\prime} \mathrm{E}\right)$, China. All plants were well-watered each day before the experiments began. At the sixth leaf stage, three drought stress treatments were imposed on the plants: (1) well-watered control treatment $(\mathrm{CK})$ : plants were watered every day to maintain soil water content (SWC) between 90 and 100\%; (2) mild-drought stress (MS): plants were irrigated to the extent of $60-70 \%$ of soil water content; (3) severe-drought stress (SS): plants were irrigated to the extent of $35-45 \%$ of soil water content. The second topmost fully expanded leaves from plants were sampled in each biological sample, immediately frozen in liquid nitrogen, and stored at $-80^{\circ} \mathrm{C}$ until analysis. Three biological replicates were set for each treatment.

Physiological parameters: The net photosynthetic rate $\left(P_{\mathrm{N}}\right)$, stomatal conductance $\left(g_{\mathrm{s}}\right)$, and intercellular $\mathrm{CO}_{2}$ concentration $\left(C_{\mathrm{i}}\right)$ of the upper second fully expanded leaves at the sixth leaf stage were determined using $L I$-Cor 6400 portable gas-exchange system (LI-COR, Huntington Beach, CA, USA). Measurements were carried out between 9:00 and 11:00 $\mathrm{h}$ under $\mathrm{CO}_{2}$ concentration of $400 \mu \mathrm{mol}$ $\mathrm{mol}^{-1}$, the PAR of $1,000 \mu \mathrm{mol}$ (photon) $\mathrm{m}^{-2} \mathrm{~s}^{-1}$, and the leaf temperature of $24 \pm 1^{\circ} \mathrm{C}$. Plant biomass production was measured after oven-drying samples at $70^{\circ} \mathrm{C}$ for $72 \mathrm{~h}$ until a constant mass was achieved and used three biological replicates as the dry mass of the whole plants.

Antioxidant activities of the upper second fully expanded leaves at the sixth leaf stage were measured, including the activities of superoxide dismutase (SOD, EC 1.15.1.1), peroxidase (POD, EC 1.11.1.7) (Luo and Huang 2012), catalase (CAT, EC 1.11.1.6), and ascorbate peroxidase (APX, EC 1.11.1.11). The measurement of APX activity was determined as described by Nakano and Asada (1981). CAT activity was performed as described by Siminis et al. (1994).

Protein extraction and trypsin digestion: The extraction of all sample proteins was performed based on the methods of Guo et al. (2017) with a slight modification. Briefly, samples were first ground in liquid nitrogen, then the tissue powder was transferred to a $5-\mathrm{mL}$ centrifuge tube and sonicated three times on ice using a high-intensity ultrasonic processor (Scientz) in lysis buffer (8 M urea, $1 \%$ Triton $X-100,10 \mathrm{mM}$ dithiothreitol, and $1 \%$ protease inhibitor cocktail). The remaining debris was removed by 
centrifugation at $12,000 \times g$ at $4^{\circ} \mathrm{C}$ for $10 \mathrm{~min}$. Finally, the supernatant was collected and the protein concertation was determined with BCA kit (Beyotime Biotechnology, Shanghai, China) according to the manufacturer's instructions.

For protein digestion, the protein solution was reduced with $5 \mathrm{mM}$ dithiothreitol for $30 \mathrm{~min}$ at $56^{\circ} \mathrm{C}$ and alkylated with $11 \mathrm{mM}$ iodoacetamide for $15 \mathrm{~min}$ at room temperature in darkness. After that, the protein sample was diluted by adding $100 \mathrm{mM}$ TEAB to urea concentration lesser than 2 M. Lastly, trypsin was added at 1:50 trypsin-to-protein mass ration for the first digestion overnight and 1:100 trypsin-to-protein mass ratio for a second $4 \mathrm{~h}$-digestion.

TMT labeling and HPLC fractionation: After trypsin digestion, the peptide was desalted by Strata X C18 SPE column (Phenomenex) and vacuum dried. The peptide was reconstituted in $0.5 \mathrm{M}$ TEAB and processed according to the manufacture's protocol for the ten-plex TMT kit (Thermo Fisher Scientific, Waltham, MA, USA). Then the sample was fractionated as previously described (Guo et al. 2017).

LC-MS/MS analysis and protein identification: Peptides were dissolved in $0.1 \%$ formic acid (solvent $\mathrm{A}$ ) and loaded directly onto a reversed-phase analytical column $(15 \mathrm{~cm}$ length, $75 \mu \mathrm{m}$ inner diameter). The gradient included an increase from 6 to $23 \%$ solvent B $(0.1 \%$ formic acid in $98 \%$ acetonitrile) over $26 \mathrm{~min}, 23$ to $35 \%$ in $8 \mathrm{~min}$, climbing to $80 \%$ in $3 \mathrm{~min}$, and then holding at $80 \%$ for the last $3 \mathrm{~min}$, all at a constant flow rate of $400 \mathrm{~nL}$ per min on an EASY-nLC 1000 UPLC system (Thermo Fisher Scientific, Waltham, MA, USA). The LC-MS/MS analysis was performed according to previously described protocols (Guo et al. 2019).

The resulting $\mathrm{MS} / \mathrm{MS}$ data were processed using the MaxQuant search engine (version 1.5.2.8.). Search parameter settings: tandem mass spectra were searched against the Uniprot_Zeamays database (99,368 sequences) concatenated with a reverse decoy database to calculate the false positive rate (FDR). Besides, a common contamination library was added to the database to eliminate the effect of contaminating proteins in the identification results. Trypsin/P was specified as the cleavage enzyme allowing up to two missing cleavages. A mass error was set as $20 \mathrm{ppm}$ in the first search and $5 \mathrm{ppm}$ in the main search for precursor ions and $0.02 \mathrm{Da}$ for fragment ions. Carbamidomethyl on Cys was specified as fixed modification and oxidation on Met was specified as variable modification. FDR was adjusted to $<1 \%$ and the minimum score for peptides was set $>40$.

Bioinformatics methods: Bioinformatic analysis was performed according to previously described protocols (Guo et al. 2019). Proteins were classified by Gene Ontology annotation (http://geneontology.org/) based on three categories: biological process, molecular function, and cellular component. KEGG database (https://www. genome.jp/kegg/) was used to identify enriched pathways. These pathways were classified into hierarchical categories according to the $K E G G$ website. All the enrichments were calculated using a two-tailed Fisher's exact test, and the GO terms and pathways with a $p$-value less than 0.05 were considered significant. The further enrichment-based clustering analysis method was detailed in Guo et al. (2019). Then, we used Wolfpsort (http://www.genscript.com/psort/ wolf_psort.html) to predict subcellular localization.

RNA extraction and quantitative real-time PCR: The samples from the control and the drought stress treatment were selected for quantitative real-time PCR (qRT-PCR) using SYBR Green I (Bio-Rad) and a CFX96 real-time PCR detection system (Bio-Rad, Hercules, USA). Specific primer sequences for real-time PCR were designed using Primer-BLAST (GenBank, NCBI). For PCR, a preliminary denaturation step at $95^{\circ} \mathrm{C}$ for $15 \mathrm{~min}$ and 40 cycles of $95^{\circ} \mathrm{C}$ for $10 \mathrm{~s}, 60^{\circ} \mathrm{C}$ for $20 \mathrm{~s}$, and $72^{\circ} \mathrm{C}$ for $30 \mathrm{~s}$. Melting curve analysis was performed to confirm the specificity of the PCR amplification. The results were calculated using the $2^{-\Delta \Delta C T}$ method (Adnan et al. 2011). Three biological replicates were performed, and gene (gene ID: GRMZM2G046804) was used as an internal control (Table 1S, supplement).

\section{Results}

Photosynthesis and antioxidant enzyme activities: Under mild-drought stress conditions, the plants showed reductions in $P_{\mathrm{N}}, g_{\mathrm{s}}$, and $C_{\mathrm{i}}$ compared to control plants (Fig. 1). The decline of the $P_{\mathrm{N}}$ and $g_{\mathrm{s}}$ accelerated at the late stage of drought exposure but $C_{\mathrm{i}}$ increased under severe drought stress. The plant biomass decreased significantly with the increasing degree of drought stress.

As shown in Fig. 2, the SOD, POD, and CAT activity increased under mild drought stress conditions and then decreased under severe drought conditions. However, APX activity gradually increased with the progress of drought stress. These results suggest that the activation of the antioxidative system may correlate with the degree of drought stress.

Identification of expressed and differentially expressed proteins (DEPs): In this study, TMT labeling and HPLC fractionation were implemented, followed by highresolution LC-MS/MS analysis and quantitative global proteome analysis. Through MaxQuant software, 80,555 spectra were matched with known spectra, corresponding to 30,496 unique peptides and 6,944 proteins. In total, 5,601 identified proteins were quantified in maize leaves. Comparative proteomic analysis was used to investigate the changes of protein profiles in leaves of Shaanke 9 under mild-drought and severe-drought stress conditions. A pairwise comparison of before and after treatments (mild stress, MS; severe stress, SS; and control, CK) was performed in Shaanke 9. DEPs were defined as proteins by using a 1.3-fold change and a $p$-value of less than 0.05 as cut-offs for significant differential expression. As shown in Fig. 3A, we found 104 DEPs between the mild-drought and control (MS/CK). Of these DEPs, 34 were upregulated whilst 70 were downregulated. 

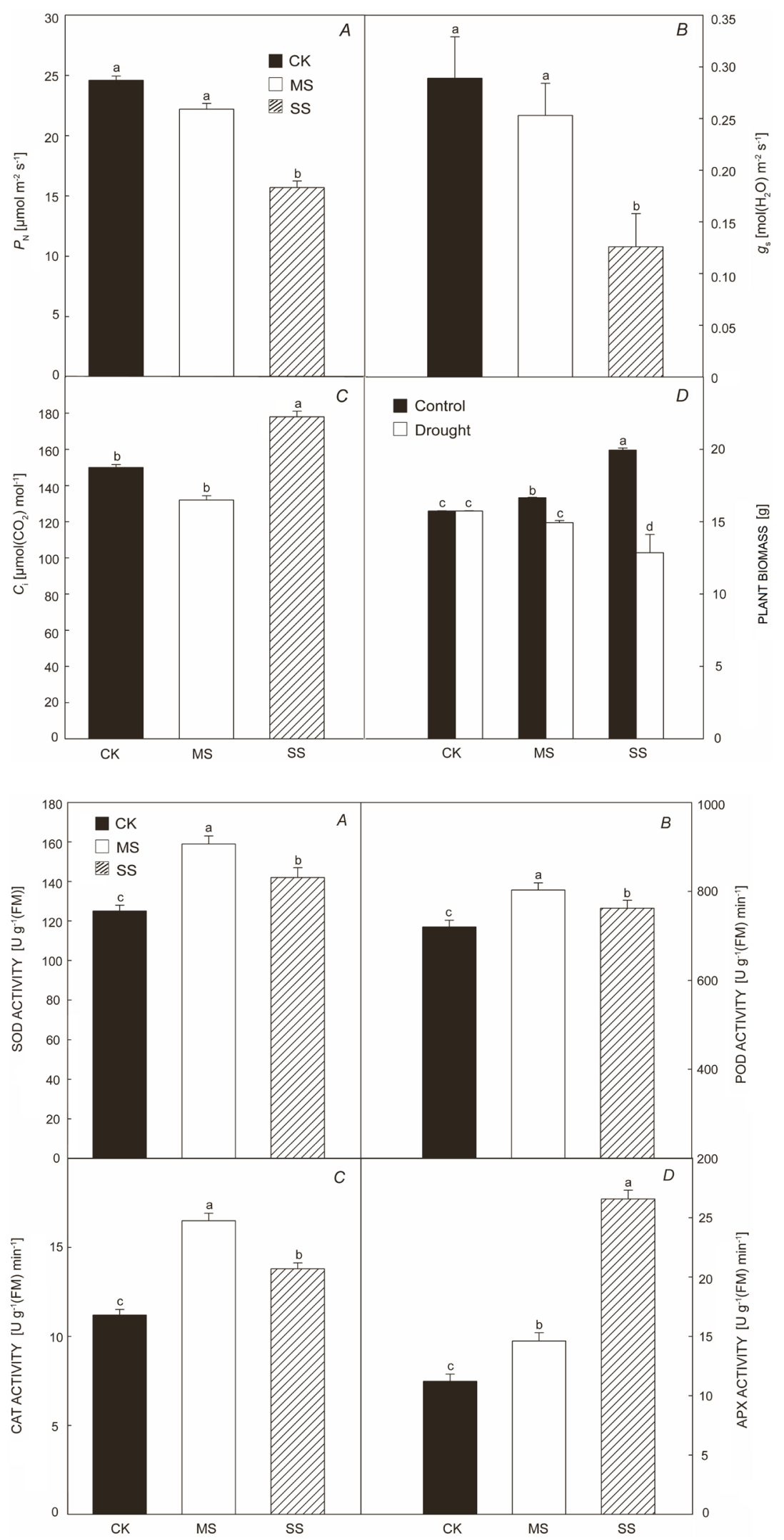

Fig. 1. Physiological parameters in maize leaves subjected to well-watered (CK), mild-drought (MS), and severe-drought (SS) stress conditions. (A) Net photosynthetic rate $\left(P_{\mathrm{N}}\right),(B)$ stomatal conductance $\left(g_{\mathrm{s}}\right)$, (C) substomatal $\mathrm{CO}_{2}$ concentration $\left(C_{\mathrm{i}}\right),(D)$ plant biomass. All data represent means \pm standard errors of three replicates. Values with different letters indicate significant difference at $p<0.05$ level between treatments according to Duncan's multiple range test.
Fig. 2. Antioxidant enzyme activities in maize leaves under well-watered (CK), mild-drought (MS), and severe-drought (SS) stress conditions. $(A)$ Superoxide dismutase (SOD), $(B)$ peroxidase (POD), $(C)$ catalase (CAT), (D) ascorbate peroxidase (APX) enzyme activity. All data represent means \pm standard errors of three replicates. Values with different letters indicate significant difference at $p<0.05$ level between treatments according to Duncan's multiple range test.
464 DEPs were observed between severe drought and control (SS/CK). Of these DEPs, 171 were upregulated and 293 were downregulated. In the severe-drought plants compared to the mild-drought group, we found 
that 252 DEPs of the 589 different expressed proteins were upregulated and 337 were downregulated. The number of downregulated proteins was higher than those of upregulated proteins. These data indicate that many more severe-drought proteins compared to mild-drought proteins were diversely expressed in response to drought. The Venn diagram shows up- and downregulated proteins

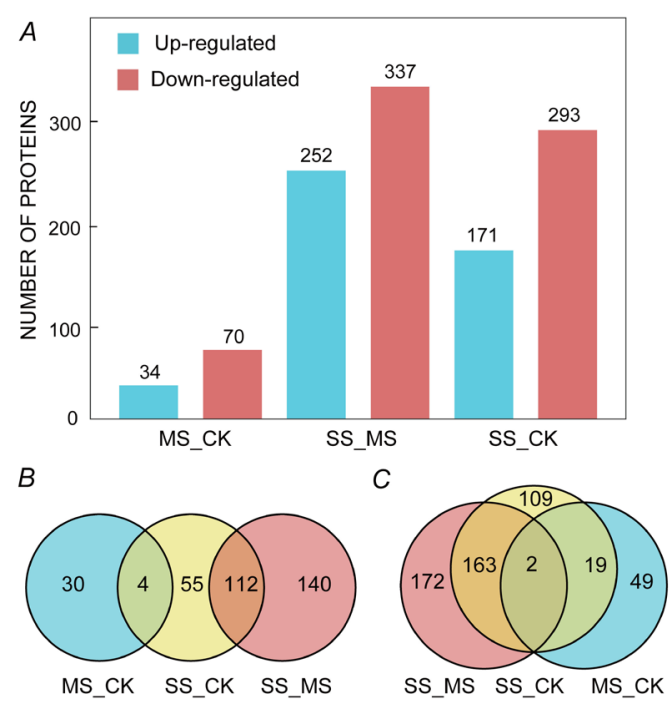

Fig. 3. (A) The number of the differentially expressed proteins in maize leaves under well-watered (control, CK), milddrought (MS), and severe-drought (SS) stress conditions. (B) Venn diagrams of upregulated differentially expressed proteins. (C) Venn diagrams of downregulated differentially expressed proteins. in comparison to mild drought, severe drought, and control, respectively (Fig. 3B,C). From the overview of DEPs in the Venn diagram, only two downregulated DEPs overlapped in all three comparison groups.

GO functional enrichment analysis of DEPs: The subcellular localization analysis (Fig. 4) showed that these proteins were mainly localized in the chloroplasts, implying the importance of chloroplast stress response. Furthermore, GO enrichment analysis was performed to reveal the function and the features of the DEPs using

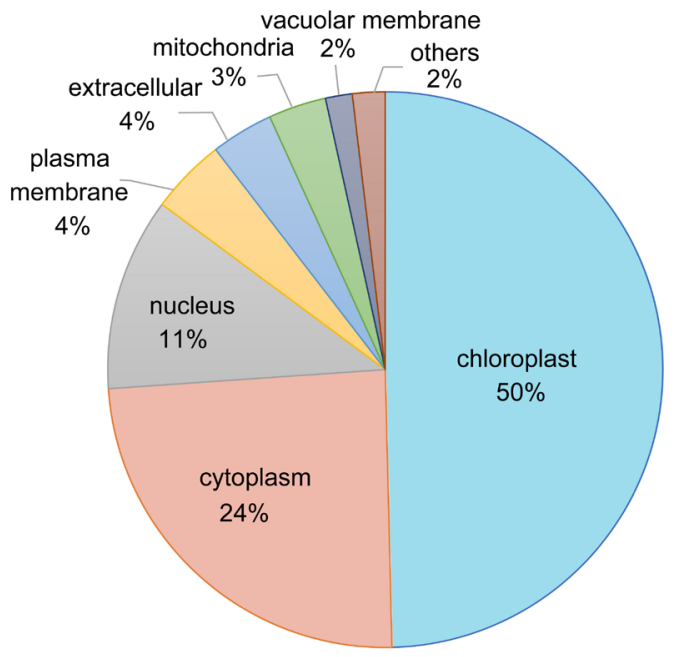

Fig. 4. Subcellular localization of proteins in maize leaves under drought stress.

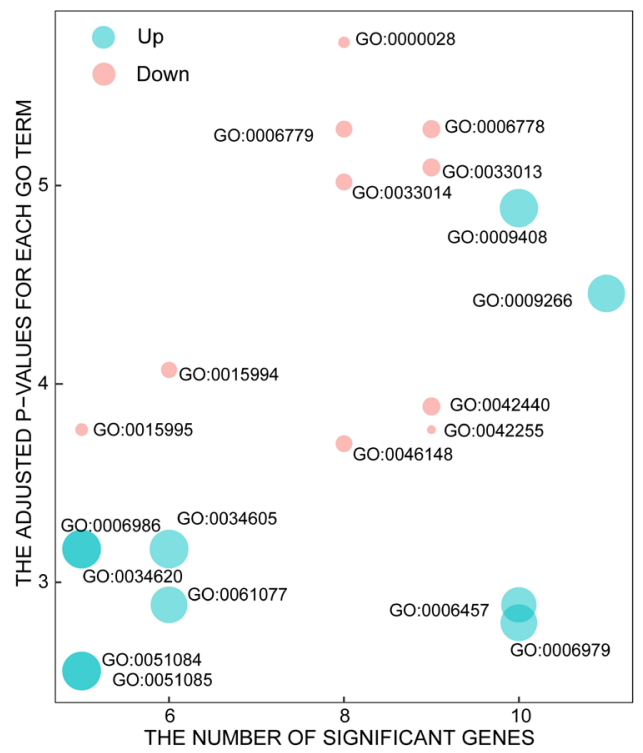

ID

\begin{tabular}{|c|c|}
\hline GO:0009408 & response to heat \\
\hline GO:0009266 & response to temperature stimulus \\
\hline GO:0034605 & cellular response to heat \\
\hline GO:0006986 & response to unfolded protein \\
\hline GO:0034620 & cellular response to unfolded protein \\
\hline GO:0061077 & chaperone-mediated protein folding \\
\hline GO:0006457 & protein folding \\
\hline GO:0006979 & response to oxidative stress \\
\hline GO:0051084 & de novo posttranslational protein folding \\
\hline GO:0051085 & chaperone mediated protein folding requiring cofactor \\
\hline GO:0000028 & ribosomal small subunit assembly \\
\hline GO:0006778 & porphyrin-containing compound metabolic process \\
\hline GO:0006779 & porphyrin-containing compound biosynthetic process \\
\hline GO:0033013 & tetrapyrrole metabolic process \\
\hline GO:0033014 & tetrapyrrole biosynthetic process \\
\hline GO:0015994 & chlorophyll metabolic process \\
\hline GO:0042440 & pigment metabolic process \\
\hline GO:0015995 & chlorophyll biosynthetic process \\
\hline GO:0042255 & ribosome assembly \\
\hline GO:0046148 & pigment biosynthetic process \\
\hline
\end{tabular}

Fig. 5. Bubble plot of significantly enriched Gene Ontology (GO) terms in the severe drought stress $v s$. control comparative datasets. The $y$-axis indicates the significance of the term $\left(-\log _{10}[\mathrm{FDR}]\right)$ and the $x$-axis indicates significant protein numbers. Bubbles indicate the GO terms, with blue indicating upregulated proteins, red indicating downregulated proteins. The bubble size indicates the expression of the proteins in the GO terms $\left\{\left[\operatorname{abs}\left(\log _{2}\right.\right.\right.$ fold change $\left.\left.)\right] \times 2\right\}$. The GO descriptions on the right are listed from the highest to the lowest significance of enrichment. 
biological process (BP) ontologies. BP analysis in SS/CK comparison group showed that the top three processes of upregulated proteins were response to heat, response to temperature stimulus, and cellular response to heat (Fig. 5), reflecting the complex nature and a degree of overlap between abiotic stress responses, while the downregulated proteins were mainly in ribosomal small subunit assembly, porphyrin-containing compound metabolic as well as a porphyrin-containing compound biosynthetic process. Our results may further suggest that the biological mechanisms of maize leaves mobilized in response to different degrees of water deficits are diverse.

KEGG pathway enrichment of DEPs: To further investigate the pathways the identified proteins are involved in, the KEGG pathways of the DEPs were performed using a $p$-value of less than 0.05 as the cut-off. We observed that the upregulated protein was considerably enriched in only one pathway (peroxisome) in the MS/CK comparison. However, the composition of the enriched KEGG pathways in the SS/CK comparison differed significantly, with porphyrin and chlorophyll metabolism, ribosome, and protein processing in the endoplasmic reticulum being the topmost enriched pathways (Fig. 6). These results show that more pathways were observed under severe drought stress.

Quantitative RT-PCR analysis: To verify the reality of our TMT sequencing data, 11 differential proteins were selected from the varying degrees of drought-responsive DEPs (Fig. 7). Of these 11 proteins, six proteins [superoxide dismutase ( $\mathrm{Cu}-\mathrm{Zn})$ 4A (P23345), catalase (K7UGM3), asparagine synthetase (A0A1D6JZD6), zeaxanthin epoxidase (A0A1D6J7P5), oxygen-evolving enhancer protein 2-1 (A0A1D6F9C2), natterin-4 (B4FHK4)] coincided well with their corresponding coding genes (Fig. $7 A-F$ ). However, the transcript expression levels of other genes were different from the relative protein levels (Fig. $7 G-K$ ), which may be due to a time delay between mRNA and proteins or posttranscriptional and transcriptional regulatory mechanisms. Overall, most of the qRT-PCR results confirmed our findings based on TMT sequencing data, as well as the expression patterns between transcript levels and corresponding protein abundance are often complex. Taken together, the overview of the metabolic processes under different drought stresses in maize leaves was shown in Fig. 8.

\section{Discussion}

Physiological responses to increasing drought stress: Many studies have observed that a drought-induced decrease in photosynthesis can be attributed to both stomatal and nonstomatal limitations (Shangguan et al. 1999). In our results, mild drought caused $P_{\mathrm{N}}, g_{\mathrm{s}}$, and $C_{\mathrm{i}}$ decrease proving the limitation of $\mathrm{CO}_{2}$ absorption which is the main factor leading to a decreased photosynthetic activity. Upon severe drought stress, nonstomatal limitations may explain the increase in $C_{\mathrm{i}}$ and the reductions of $P_{\mathrm{N}}$. Further, the reduction in plant biomass indicates that drought stress inhibited the growth of maize seedlings.

Plants can activate the enzymatic antioxidant system, which operates with the sequential and simultaneous action of several enzymes including SOD, POD, CAT, and APX (Hegedüs et al. 2001). Our results demonstrated that antioxidant enzyme activities changed under different degrees of drought stress. The increase of SOD, POD, and CAT under mild drought stress shows that these enzymes constitute the first line of cell protection from oxidative damage. However, the activities of the above-mentioned antioxidants were decreased and APX served as an antioxidative defense system under severe drought stress, consistent with previous findings in Pteroceltis tatarinowii (Liu et al. 2011).

Proteins involved in antioxidant system under mild drought stress: The abundances of defense response proteins changed under drought stress. Redox balance plays an important role in protecting plants from droughtinduced oxidative stress that is essential for stress adaptation (Chaves and Oliveira 2004). In our study, biosynthesis-related proteins of antioxidant enzymes

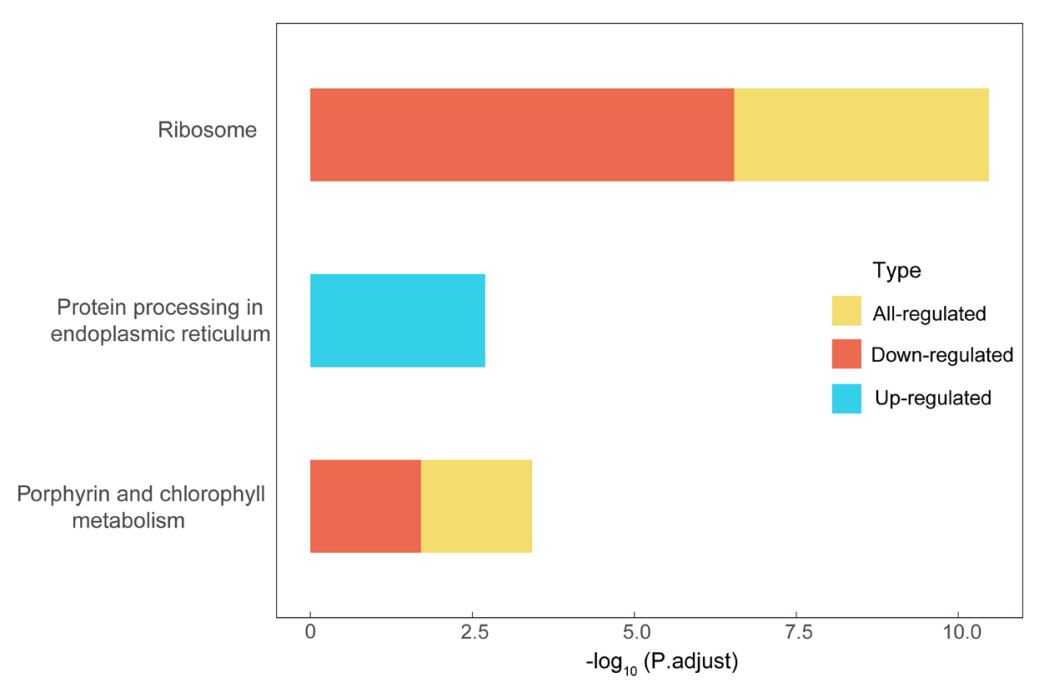

Fig. 6. The bar plot showing the enrichments scores $\left(-\log _{10}\right.$ [P.adjust]) of the significant enrichments KEGG pathways under severe drought stress $v s$. control conditions. 


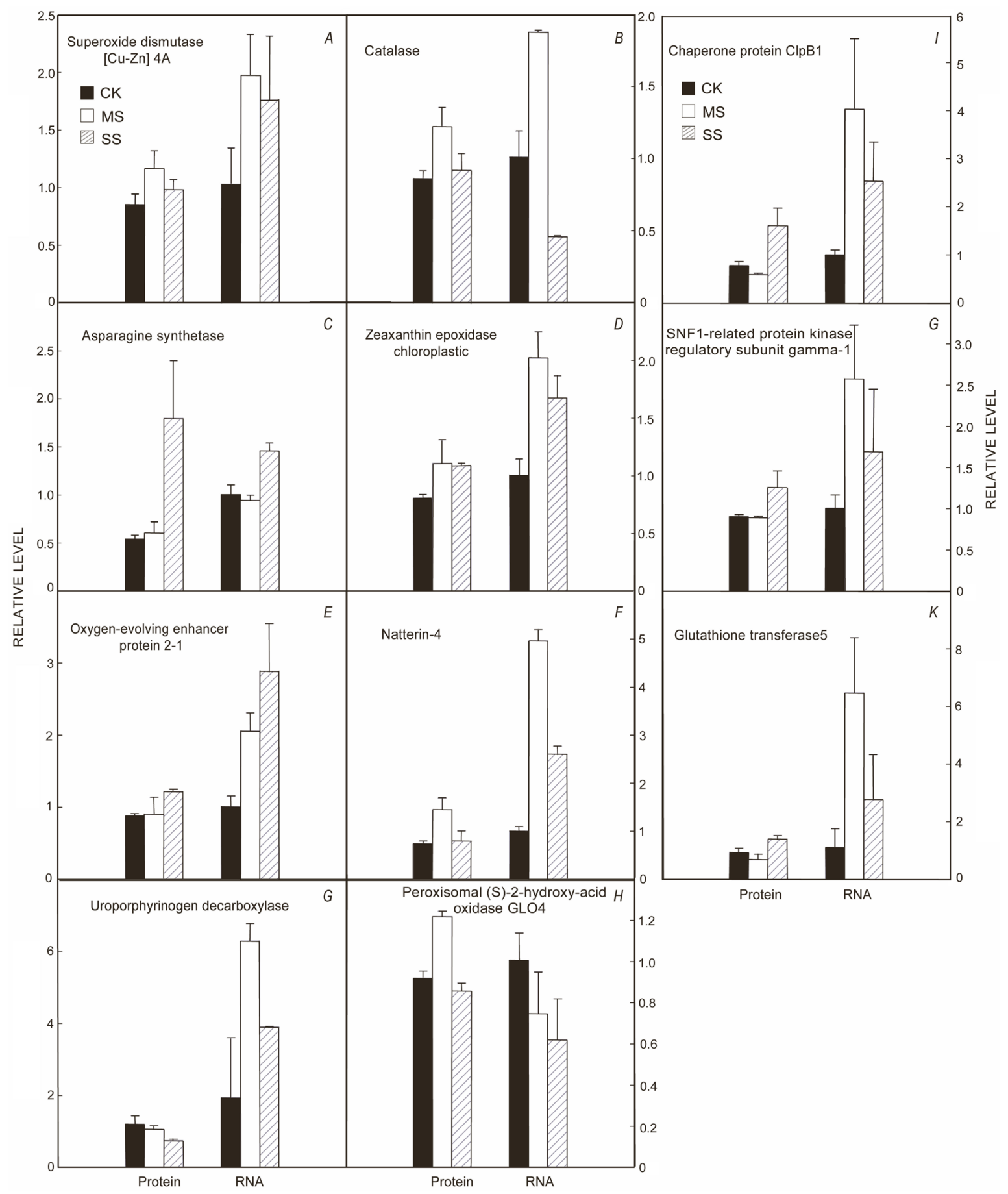

Fig. 7. Analysis of proteomic results by quantitative real-time PCR (qRT-PCR). The bars represent fold change of mRNA and protein abundances in maize leaves under well-watered (CK), mild-drought (MS), and severe-drought (SS) stress conditions, respectively.

including SOD, POD, GST, APX, and CAT were upregulated in maize leaves under mild drought conditions (Table 2S, supplement), suggesting that mild drought induces the antioxidant defense system to scavenge ROS and decrease oxidative damage in maize seedling leaves, possibly providing a favorable environment for growth and development processes. This is consistent with the results of SOD, POD, and CAT. A previous study (Kushwaha et al. 2009) observed that all the cystathionine $\beta$-synthase (CBS) domain-containing proteins were upregulated under drought stress and might play an important role in stress response/tolerance of Arabidopsis under various stress conditions. Our results found the cystathionine $\beta$-synthase (CBS) domain-containing protein showed a significant 


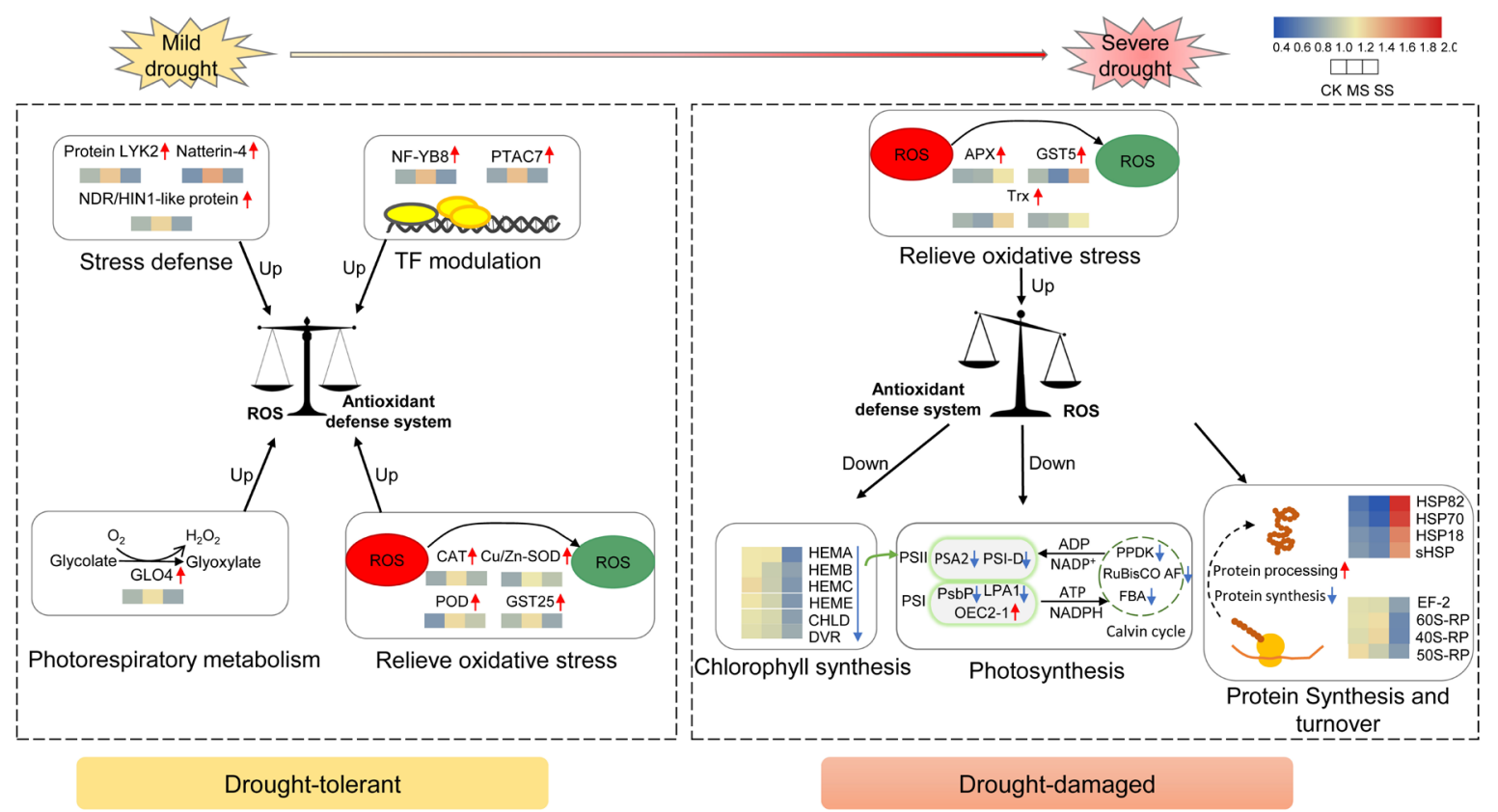

Fig. 8. Drought stress-induced metabolic changes in maize seedling leaves. NF-YB8 - nuclear transcription factor Y subunit B-8; PTAC7 - protein PLASTID TRANSCRIPTIONALLY ACTIVE 7; GLO4 - peroxisomal (S)-2-hydroxy-acid oxidase GLO4; $\mathrm{Cu} / \mathrm{Zn}$-SOD - superoxide dismutase [Cu-Zn] 4A; CAT - catalase/hydrogen peroxidase; POD - peroxidase; GST25 - glutathione transferase25; APX - APx1-cytosolic ascorbate peroxidase; GST5 - glutathione transferase5; Trx - thioredoxin family protein; HEMA - glutamyl-tRNA reductase; HEMB - delta-aminolevulinic acid dehydratase; HEMC - porphobilinogen deaminase; HEME uroporphyrinogen decarboxylase; CHLD - magnesium-chelatase subunit ChlH, chloroplastic; DVR - divinyl reductase; PSA2 protein PHOTOSYSTEM I ASSEMBLY 2; PSI-D - photosystem I subunit D1; PsbP - PsbP domain-containing protein 5, chloroplastic; LPA1 - protein LOW PSII ACCUMULATION 1, chloroplastic; OEC2-1 - oxygen-evolving enhancer protein 2-1, chloroplastic; PPDK - pyruvate, phosphate dikinase; RuBisCO AF - Rubisco accumulation factor2; FBA - fructose-bisphosphate aldolase; HSP82 - heat shock protein 82; HSP70 - heat shock protein 70; HSP18 - heat shock protein 18; sHSP - small heat shock protein; EF-2 - elongation factor 2; 60S-RP - 60S ribosomal protein L27; 40S-RP - 40S ribosomal protein S12; 50S-RP - 50S ribosomal protein L32, chloroplastic. The upregulated proteins are marked and the downregulated are marked under mild- and severe drought stress, respectively.

increase and then decreased sharply with the duration of dehydration, this may be the result of biological processes regulating in response to varying degrees of drought stress.

Proteins involved in photorespiration under mild drought stress: It is well known that photosynthesis is protected from photoinhibition by photorespiration. Under water-deficit conditions, the decrease of stomatal conductance reduces the content of intercellular $\mathrm{CO}_{2}$ in leaves, thereby promoting an increase in photorespiration. Besides, the photosynthetic electron transport chain is a major source of ROS in plant cells, and the process of photorespiration helps minimize ROS production by dissipating excess reducing equivalents as well as energy (Voss et al. 2013). Glycolate oxidase (GLO) is a key enzyme in photorespiratory metabolism, which catalyzes the oxidation of glycolate to acetaldehyde and produces $\mathrm{H}_{2} \mathrm{O}_{2}$ (Zhang et al. 2012). The upregulated expression of glycolate oxidase $\mathrm{GLO}_{4}$ in our results (Table $2 \mathrm{~S}$ ) indicates that photorespiration acts as an important energy dissipation pathway for protection of the photosynthetic apparatus from photodamage during mild drought stress, while the $\mathrm{GLO}_{4}$ was downregulated under severe drought conditions. In response to mild drought stress, increased photorespiration should be enough to dissipate excess light as photosynthesis decreases. Under severe drought conditions, however, photorespiration reduced possibly due to the decrease of ribulose-1,5-bisphosphate availability and/or Rubisco activity (Flexas and Medrano 2002).

Proteins involved in other biological processes under mild drought stress: Transcription factors (TFs) play a major role in gene regulation under drought stress. Our findings verified that nuclear transcription factor $Y$ subunit B-8 (NF-YB8) and protein plastid transcriptionally active 7 (PTAC7) were upregulated in response to mild-drought stress (Table 2S). NF-Y is a ubiquitous transcription factor and overexpression of NF-YB3 in wheat increases chlorophyll content and rate of photosynthesis under normal conditions (Stephenson et al. 2011). Research indicates that PTAC7 is involved in the function of regulating chloroplast gene expression (Pfalz et al. 2006). Upregulation of these two transcription factors might be a potent tool for studying the mechanism of mild drought stress. Besides, the abundances of the unknown functional 
protein natterin- 4 were enhanced in stressed leaves. Similar proteomic results showed that the abundance of natterin-4 was enhanced in maize leaves under polyethylene glycol (PEG)-stimulated water deficiency (Shao et al. 2015). These consistent results indicate that natterin- 4 responds to water deficiency.

Proteins involved in protein metabolism under severe drought stress: The abundances of transcription-related proteins, ribosomal proteins, protein chaperones, and proteases were all changed under severe drought stress (Table 3S, supplement). G2-like transcription factor helps coregulate and synchronize the expression of a suite of nucleus photosynthetic genes and thus acts to optimize photosynthetic capacity under varying environmental and developmental conditions (Waters et al. 2009). The upregulation of the G2-like transcription factor in our results suggested this gene might play vital roles in the stress response. Three upregulated proteins functioning in protein biosynthesis included eukaryotic translation initiation factor 4F (eIF-4F), putative translation elongation factor, and $40 \mathrm{~S}$ ribosomal protein $\mathrm{S} 7$, which are directly involved in the initiation and elongation of the newly growing peptide chains. The other identified proteins, such as eIF-3D, eIF-5C, and most ribosome proteins, were downregulated under severe drought stress. Similar results were found in $\mathrm{H}_{2} \mathrm{O}_{2}$-treated rice seedling leaves (Wan and Liu 2008). Remarkably, the opposite change patterns of ribosomal proteins may implicate their different roles in the drought-treated maize leaves. Moreover, most ribosomal proteins are remarkably decreased suggesting that protein biosynthesis may be inhibited under severe stress conditions. Besides, most heat shock proteins (HSPs) including HSP70 family, HSP90 family, and the small HSP family increased under severe drought stress. Although each family of these HSPs has a unique mechanism (Al-Whaibi 2011), major HSPs have some kind of related roles in solving the problem of misfolding and aggregation, as well as a role as chaperones. These data suggest that maintaining correct protein folding is important for leaves to cope with severe drought stress. Serine carboxypeptidase generally plays a role in protein/ peptide processing and degradation. For the ubiquitin/26S proteasome system, E3 ubiquitin ligases were positively related to plant drought stress (Bae et al. 2011). Thus, the upregulation of these proteins indicates that protein degradation is enhanced in severe drought-treated maize seedling leaves. However, some downregulated proteins associated with proteases may occur due to increased sensitivity of leaves to severe drought stress. Taken together, the regulated expression response patterns of all the proteins mirror the fact that the inhibition of novel protein biosynthesis and the enhancement of protein folding as well as protein degradation are required for the survival and growth of maize plant under severe drought stress.

Proteins involved in photosynthesis and chlorophyll metabolism under severe drought stress: Photosynthesis can normally be divided into two processes: the light reaction and the carbon fixation reaction. Serious drought stress decreased six DEPs which were involved in photoreaction, i.e., protein PSI assembly 2, PSI subunit D1, PSI reaction center subunit V, PsbP domaincontaining protein 5 (PPD5), protein curvature thylakoid $1 \mathrm{~B}$, and chlorophyll $a / b$ binding protein (Table $3 \mathrm{~S}$ ). The decrease of PSI-related DEPs showed that severe drought stress injured PSI protein quantity and integrity. PSII subunit $\mathrm{P}(\mathrm{PsbP})$ protein is part of the oxygenevolution complex (OEC) of PSII and stabilizes Mn clusters to ensure the normal function and high efficiency of PSII (Ifuku et al. 2005). Reduced levels of the OEC-related protein PsbP are associated with reduced photosynthetic capacity. LOW PSII ACCUMULATION 1 is engaged in the efficient assembly of the PSII complex (Peng et al. 2006). Expression of LOW PSII ACCUMULATION 1 increased under severe drought stress which may be due to the damage of PSII. Whereas, the increase of oxygen-evolving enhancer protein 2-1 (a component of the OEC complex) located in PSII also suggested that more oxygen is produced to enhance the stability of PSII under severe drought conditions. The chlorophyll $a / b$ binding protein is a member of the lightharvesting complex (LHC) that captures external energy and transmits it to the photosystem for photosynthesis. These results implied that severe drought stress inhibited light harvesting and electron transfers due to PSII structural damages. The abundance of proteins involved in the Calvin cycle, including pyruvate, phosphate dikinase (PPDK), Rubisco accumulation factor 2, and fructose-bisphosphate aldolase (chloroplast-localized enzyme), decreased. All these enzymes play crucial roles in the Calvin cycle of $\mathrm{C}_{4}$ plants. Besides, the decrease in these enzymes indicates that severe drought leads to reduced $\mathrm{CO}_{2}$ fixation capacity and photosynthetic capacity. Two proteins associated with carotenoid biosynthesis were identified in our study. We found the upregulation of lycopene beta/epsilon cyclase protein and zeaxanthin epoxidase under severe drought conditions, which implied that carotenoids could protect plants from oxidative damage caused by drought. Meanwhile, the xanthophyll-circulating substances are the synthetic precursors of the key hormone abscisic acid (ABA).

Chlorophyll $(\mathrm{Chl})$ plays a vital role in the process of light harvesting and energy transduction in photosynthesis (Tripathy and Pattanayak 2012). Also, Chl is required for the correct folding, assembly, and insertion into thylakoid membranes of photosynthetic proteins (Kim et al. 1994). The most enrichment pathways in biological processes and KEGG pathways related to severe drought stress in maize seedlings were porphyrin and $\mathrm{Chl}$ metabolism in the current study. The expression of eleven genes related to the Chl biosynthesis pathway was changed (Table 3S). Most Chl biosynthetic proteins decreased after severe drought indicating that $\mathrm{Chl}$ biosynthesis was inhibited in droughtstressed leaves. Chl loss is a negative consequence of stress, disrupting the structure and function of chloroplast. On the other hand, Chl loss reduces the amount of light absorption by leaves and also reduces the possibility of the formation of activated oxygen to further destroy the 
photosynthetic machinery (Munné-Bosch and Alegre 2000). These findings suggested that the synthesis of Chl was reduced under severe drought conditions.

In addition to photosynthesis-related proteins, the abundance of proteins related to carbohydrate and energy metabolism also changed. This includes six proteins involved in glycolysis and the TCA cycle, such as pyruvate kinase, glycerolphosphate mutase, ATP-dependent 6-phosphofructokinase, and isocitrate dehydrogenase [NAD] regulatory subunit 3. Two necessary proteins related to the pentose phosphate pathway (PPP) increased, including glucose-6-phosphate-1-dehydrogenase, and pyrophosphate-fructose 6-phosphate 1-phosphotransferase subunit beta. Another upregulated protein involved in the mitochondrial electron transport chain was cytochrome $b c 1$ complex subunit 7 . The increase of TCA and PPP may provide energy during the activation of stress defenses, especially when the photosynthesis was inhibited. Their changes imply that plants may have the ability to enhance energy production to inhibit drought damage.

Proteins involved in other biological processes under severe drought stress: Large number of signal transduction-related proteins were identified to be differentially expressed in the drought-stressed leaves (Table 3S). These proteins mainly comprise protein kinase, $\mathrm{G}$ protein, $\mathrm{Ca}^{2+}$ signaling, and ABA-responsive, and stress ripeninginduced proteins. Besides, other hormonal signals, such as ethylene and cytokinins, were also changed under severe drought stress. Our results indicated that most protein kinases (e.g., mitogen-activated protein kinase and SNF1related protein kinase) and MAPK signaling proteins were upregulated, especially the Ras-related protein RABD1, which increased 2.415-fold. Meanwhile, drought also induced significant upregulation of phytohormone ethylene (ERF) and ABA-related proteins, but the proteins involved in cytokinin biosynthesis showed the opposite expression. Thus, we can speculate that signaling pathways are interconnected to constitute the networks that lead to various plant responses under severe drought stress. The specific signal perception and conduction pathways need further study. Furthermore, the cytoskeleton-related protein tubulin beta-1 chain, which is an essential component of the microtubules, decreased in response to severe drought stress. The decrease of the cytoskeleton proteins showed that cell growth is suppressed during severe drought stress.

Conclusions: Combined with physiology, proteomics, and gene expression data, our results demonstrate that activated enzymic antioxidants and photorespiration are important adaptive responses under mild drought stress, while severe drought damaged photosynthesis and the equilibrium of protein metabolism in maize leaves. It is expected that further desiccation would result in irreversible damage to maize. Furthermore, our results have shown that divergent antioxidative systems exist between mild and severe drought stress. Our findings depict a panoramic view of the adaptation and response processes occurring in the drought-treated maize seedling leaves. Based on our findings, we proposed a molecular model during increasing drought stress in maize seedling leaves (Fig. 8). Clear interpretation of these identified proteins in plant drought response needs to be further investigated.

\section{References}

Adnan M., Morton G., Hadi S.: Analysis of $r p o S$ and bolA gene expression under various stress-induced environments in planktonic and biofilm phase using 2(- $\Delta \Delta \mathrm{CT})$ method. - Mol. Cell. Biochem. 357: 275-282, 2011.

Al-Whaibi M.H.: Plant heat-shock proteins: A mini review. J. King Saud Univ. Sci. 23: 139-150, 2011.

Anjum S.A., Xie X.Y., Wang L.C. et al.: Morphological, physiological and biochemical responses of plants to drought stress. - Afr. J. Agr. Res. 6: 2026-2032, 2011.

Bae H., Kim S.K., Cho S.K. et al.: Overexpression of OsRDCP1, a rice RING domain-containing E3 ubiquitin ligase, increased tolerance to drought stress in rice (Oryza sativa L.). - Plant Sci. 180: 775-782, 2011.

Chaves M.M., Oliveira M.M.: Mechanisms underlying plant resilience to water deficits: prospects for water-saving agriculture. - J. Exp. Bot. 55: 2365-2384, 2004.

Chen C., Cao Q., Jiang Q. et al.: Comparative transcriptome analysis reveals gene network regulating cadmium uptake and translocation in peanut roots under iron deficiency. - BMC Plant Biol. 19: 35, 2019.

Cui G., Zhao Y., Zhang J. et al.: Proteomic analysis of the similarities and differences of soil drought and polyethylene glycol stress responses in wheat (Triticum aestivum L.). Plant Mol. Biol. 100: 391-410, 2019.

Dong T., Zhu M., Yu J. et al.: RNA-Seq and iTRAQ reveal multiple pathways involved in storage root formation and development in sweet potato (Ipomoea batatas L.). - BMC Plant Biol. 19: 136, 2019.

Flexas J., Medrano H.: Energy dissipation in $\mathrm{C}_{3}$ plants under drought. - Funct. Plant Biol. 29: 1209-1215, 2002.

Guo H., Guo H., Zhang L. et al.: Dynamic TMT-based quantitative proteomics analysis of critical initiation process of totipotency during cotton somatic embryogenesis transdifferentiation. - Int. J. Mol. Sci. 20: 1691, 2019.

Guo J.H., Liu J.X., Wei Q. et al.: Proteomes and ubiquitylomes analysis reveals the involvement of ubiquitination in protein degradation in petunias. - Plant Physiol. 173: 668-687, 2017.

Habibi G.: Effects of mild and severe drought stress on the biomass, phenolic compounds production and photochemical activity of Aloe vera (L.) Burm.f. - Acta Agr. Slov. 111: 463476, 2018 .

Hegedüs A., Erdei S., Horváth G.: Comparative studies of $\mathrm{H}_{2} \mathrm{O}_{2}$ detoxifying enzymes in green and greening barley seedlings under cadmium stress. - Plant Sci. 160: 1085-1093, 2001.

Huang L., Zhang F., Zhang F. et al.: Comparative transcriptome sequencing of tolerant rice introgression line and its parents in response to drought stress. - BMC Genomics 15: 1026, 2014.

Ifuku K., Yamamoto Y., Ono T.A. et al.: PsbP protein, but not PsbQ protein, is essential for the regulation and stabilization of photosystem II in higher plants. - Plant Physiol. 139: 11751184, 2005.

Jiang Z., Jin F., Shan X., Li J.: iTRAQ-based proteomic analysis reveals several strategies to cope with drought stress in maize seedlings. - Int. J. Mol. Sci. 20: 5956, 2019.

Jogaiah S., Govind S.R., Tran L.-S.P.: Systems biology-based approaches toward understanding drought tolerance in food crops. - Crit. Rev. Biotechnol. 33: 23-39, 2013.

Kim J., Eichacker L.A., Rudiger W., Mullet J.E.: Chlorophyll regulates accumulation of the plastid-encoded chlorophyll 
proteins P700 and D1 by increasing apoprotein stability. Plant Physiol. 104: 907-916, 1994.

Kim S.G., Lee J.S., Kim J.T. et al.: Physiological and proteomic analysis of the response to drought stress in an inbred Korean maize line. - Plant Omics 8: 159-168, 2015.

Kushwaha H.R., Singh A.K., Sopory S.K. et al.: Genome wide expression analysis of CBS domain containing proteins in Arabidopsis thaliana (L.) Heynh and Oryza sativa L. reveals their developmental and stress regulation. - BMC Genomics 10: $200,2009$.

Liu C., Liu Y., Guo K. et al.: Effect of drought on pigments, osmotic adjustment and antioxidant enzymes in six woody plant species in karst habitats of southwestern China. Environ. Exp. Bot. 71: 174-183, 2011.

Liu J., Guo Y.Y., Bai Y.W. et al.: Effects of drought stress on the photosynthesis in maize. - Russ. J. Plant Physl+ 65: 849-856, 2018b.

Liu W., Xu W., Li L. et al.: iTRAQ-based quantitative tissue proteomic analysis of differentially expressed proteins (DEPs) in non-transgenic and transgenic soybean seeds. - Sci. Rep.-UK 8: 17681, 2018 a.

Luo X., Huang Q.: Studies on the cold resistance of cassava. J. Agr. Sci. 4: 104-119, 2012.

Munné-Bosch S., Alegre L.: Changes in carotenoids, tocopherols and diterpenes during drought and recovery, and the biological significance of chlorophyll loss in Rosmarinus officinalis plants. - Planta 210: 925-931, 2000.

Nakano Y., Asada K.: Hydrogen peroxide is scavenged by ascorbate-specific peroxidase in spinach chloroplasts. - Plant Cell Physiol. 22: 867-880, 1981.

Peng L., Ma J., Chi W. et al.: LOW PSII ACCUMULATION1 is involved in efficient assembly of photosystem II in Arabidopsis thaliana. - Plant Cell 18: 955-969, 2006.

Pfalz J., Liere K., Kandlbinder A. et al:: PTAC2, -6, and -12 are components of the transcriptionally active plastid chromosome that are required for plastid gene expression. Plant Cell 18: 176-197, 2006.

Salehi-Lisar S.Y., Bakhshayeshan-Agdam H.: Drought stress in plants: causes, consequences, and tolerance. - In: Hossain M.A., Wani S.H., Bhattacharjee S. (ed.): Drought Stress Tolerance in Plants. Vol. 1. Pp. 1-16. Springer, Cham 2016.

Shangguan Z., Shao M., Dyckmans J.: Interaction of osmotic adjustment and photosynthesis in winter wheat under soil drought. - J. Plant Physiol. 154: 753-758, 1999.

Shao R., Xin L., Mao J. et al.: Physiological, ultrastructural and proteomic responses in the leaf of maize seedlings to polyethylene glycol-stimulated severe water deficiency. - Int J. Mol. Sci. 16: 21606-21625, 2015.

Siminis C.I., Kanellis A.K., Roubelakis-Angelakis K.A.: Catalase is differentially expressed in dividing and nondividing protoplasts. - Plant Physiol. 105: 1375-1383, 1994.

Solis J., Baisakh N., Brandt S.R. et al.: Transcriptome profiling of beach morning glory (Ipomoea imperati) under salinity and its comparative analysis with sweetpotato. - PLoS ONE 11: e0147398, 2016.

Stephenson T.J., McIntyre C.L., Collet C., Xue G.P.: TaNFYB3 is involved in the regulation of photosynthesis genes in Triticum aestivum. - Funct. Integr. Genomic. 11: 327-340, 2011.

Tripathy B.C., Pattanayak G.K.: Chlorophyll biosynthesis in higher plants. - In: Eaton-Rye J., Tripathy B.C., Sharkey T. (ed.): Photosynthesis. Advances in Photosynthesis and Respiration. Pp. 63-94. Springer, Dordrecht 2012.

Voss I., Sunil B., Scheibe R., Raghavendra A.S.: Emerging concept for the role of photorespiration as an important part of abiotic stress response. - Plant Biol. 15: 713-722, 2013.

Wan X.Y., Liu J.Y.: Comparative proteomics analysis reveals an intimate protein network provoked by hydrogen peroxide stress in rice seedling leaves. - Mol. Cell. Proteomics 7: 1469$1488,2008$.

Waters M.T., Wang P., Korkaric M. et al.: GLK transcription factors coordinate expression of the photosynthetic apparatus in Arabidopsis. - Plant Cell 21: 1109-1128, 2009.

Wu Y., Mirzaei M., Atwell B.J., Haynes P.A.: Label-free and isobaric tandem mass tag (TMT) multiplexed quantitative proteomic data of two contrasting rice cultivars exposed to drought stress and recovery. - Data Brief 22: 697-702, 2019.

Zhang Z., Lu Y., Zhai L. et al.: Glycolate oxidase isozymes are coordinately controlled by $G L O 1$ and GLO4 in rice. - PLOS ONE 7: e39658, 2012.

Zheng H., Yang Z., Wang W. et al.: Transcriptome analysis of maize inbred lines differing in drought tolerance provides novel insights into the molecular mechanisms of drought responses in roots. - Plant Physiol. Bioch. 149: 11-26, 2020.

Zhou S., Li M., Guan Q. et al.: Physiological and proteome analysis suggest critical roles for the photosynthetic system for high water-use efficiency under drought stress in Malus. Plant Sci. 236: 44-60, 2015.

Zhu J.K.: Salt and drought stress signal transduction in plants. Annu. Rev. Plant Biol. 53: 247-273, 2002.

Zhu J.K.: Abiotic stress signaling and responses in plants. - Cell 167: 313-324, 2016.

(C) The authors. This is an open access article distributed under the terms of the Creative Commons BY-NC-ND Licence. 